\title{
Hip Dislocation
}

\section{Are Hip Precautions Necessary in Anterior Approaches?}

\author{
Camilo Restrepo MD, S. M. Javad Mortazavi MD, \\ Justin Brothers BS, Javad Parvizi MD FRCS, \\ Richard H. Rothman MD, PhD
}

Published online: 13 November 2010

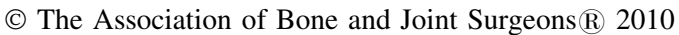

\begin{abstract}
Background In 2005, we reported removal of functional restriction after primary THA performed through the anterolateral approach did not increase the incidence of dislocation.

Questions/purposes To develop a current practice guideline, we evaluated the incidence of early dislocation after primary THA after implementation of a no-restriction protocol.

Methods Between January 2005 and December 2007, 2532 patients ( 2764 hips; 1541 women, 1223 men; mean age, 63.2 years [28-98 years]) underwent primary THA at our institution. Bilateral THA was performed in 232 patients (464 hips). The direct anterior or anterolateral approach was used in all patients. Femoral head size was 28,32 , or $36 \mathrm{~mm}$. Patients were given no traditional functional restrictions postoperatively, such as use of elevated seats, abduction pillows, and restriction from driving. All patients received
\end{abstract}

Javad Parvizi is a consultant for Stryker Orthopaedics (Mahwah, NJ) and has Intellectual Properties on SmarTech (Philadelphia, PA);

Richard H. Rothman is a consultant for and receives royalties from Stryker Orthopaedics.

Each author certifies that his/her institution has approved the human protocol for this investigation and that all investigations were conducted in conformity with ethical principles of research.

C. Restrepo, S. M. J. Mortazavi, J. Brothers, J. Parvizi,

R. H. Rothman ( $\square)$

The Rothman Institute of Orthopaedics, Thomas Jefferson

University Hospital, 925 Chestnut Street, 5th Floor,

Philadelphia, PA 19107, USA

e-mail: research@rothmaninstitute.com;

camilo.restrepo@rothmaninstitute.com

S. M. J. Mortazavi

Department of Orthopaedic Surgery, Tehran University for Medical Sciences, Tehran, Iran standard care at the judgment of the attending surgeon. One hundred forty-six patients missed followup appointments despite efforts to be contacted by telephone. The remaining 2386 of 2532 patients (94\%) had a minimum followup of 6 months (mean, 14.2 months; range, 6-34 months).

Results Four known dislocations occurred in the followed cohort of 2386 patients with 2612 hips $(0.15 \%)$ at a mean of 5 days (3-12 days) postoperatively, none related to high-impact trauma. One dislocation occurred in a patient with a history of developmental dysplasia of the hip, two dislocations occurred while at the toilet (one with a previous hip fracture treated with a modular system), and one dislocation was idiopathic.

Conclusions We confirmed a low incidence of dislocation after primary THA in the absence of early postoperative restrictions. We conclude a no-restriction protocol does not increase the incidence of early dislocation after primary THA.

Level of Evidence Level II, therapeutic study. See the Guidelines for Authors for a complete description of levels of evidence.

\section{Introduction}

Early postoperative dislocation is a rare yet devastating complication after THA for the patient and the surgeon. The incidence of dislocation reportedly ranges from less than $1 \%$ to greater than $15 \%$ [1, 3, 5, 16, 21]. This wide variation in incidence is undoubtedly related to many factors, including a different cohort of patients with different characteristics, different surgical techniques, different implant design, and variable clinical followup.

In most series, the majority of initial dislocations occur early. In one report of 6774 patients from 1981, 
approximately $60 \%$ to $70 \%$ reported within the first 4 to 6 weeks after the operative procedure [1]. Some series reported a lower percentage (39\%) [21] of early compared with late dislocations probably due to longer followup and identification of more late dislocations. Nonetheless, there is a high-risk period for occurrence of dislocation in the first months after THA. Therefore, there was a common trend for surgeons to apply historically described patient education and functional restrictions to help reduce the rate of postoperative dislocations in patients undergoing THA by preventing extremes of ROM [2]. However, controlling for other more important factors, including preoperative planning, correct prosthesis selection, surgical technique, and accurate component positioning, may alleviate the need for these restrictions.

In 2002, Talbot et al. [19] suggested eliminating functional restrictions and restricting patients' postoperative mobilization was unnecessary. Our institution reported in 2005 removal of functional restriction after primary THA performed through the anterolateral approach did not increase the incidence of dislocation [14]. Although that study was a randomized controlled trial, the number of patients in each branch was relatively small with regard to low incidence of dislocation (less than 1\%). Since that time, we have abandoned the use of patient restrictions after primary THA.

To develop current practice guidelines, we needed to assess this protocol in a large cohort of patients who received a primary THA. We therefore evaluated early dislocation rates in a larger, uniformly nonrestricted patient population.

\section{Patients and Methods}

All patients undergoing primary THA between January 1, 2005, and December 31, 2007, at our institution were included in this study. Patients with a history of surgery on the contralateral hip, hyperflexibilty syndrome, developmental dysplasia of the hip (DDH), and neuromuscular disease were also included. Revision and conversion surgeries were not included as this study was designed to evaluate primary THA.

The no-restriction protocol used in our institution is applied to all patients undergoing primary THA. It entitles patients to move their operated hip through ROM based on the patient's comfort (including flexion and adduction) starting right after surgery. Therefore, we did not apply abduction pillows in the operating room; we did not require patients to use pillows to maintain abduction while in bed, elevated toilet seats, and elevated seats at the hospital, rehabilitation facilities, and home; and we did not prevent them from sleeping on the operated side, from driving, and from being passengers in an automobile.
The study group comprised 2532 patients (2764 hips), including 1541 women and 1223 men with a mean age of 63.2 years (range, 28-98 years). The mean body mass index (BMI) was $29.9 \mathrm{~kg} / \mathrm{m}^{2}$ (range, $16.5-58.2 \mathrm{~kg} / \mathrm{m}^{2}$ ) and $31.0 \mathrm{~kg} / \mathrm{m}^{2}$ (range, $13.7-54.0 \mathrm{~kg} / \mathrm{m}^{2}$ ) for men and women, respectively. The ages and $\mathrm{BMI}$ of the patient population followed a normal curve. Bilateral THA was performed in 232 patients (464 hips), with 193 patients simultaneous and 39 patients staged. One hundred forty-six patients (152 hips) were lost to followup at the office for their 6-month postoperative visit. Despite all efforts, we were unable to contact them by telephone or other means to complete evaluation. Thus, 94\% (2386 of 2532 patients with 2612 hips) were followed for the minimum 6 months (mean, 14.2 months; range, 6-34 months) postoperatively.

Most patients underwent neuraxial anesthesia, with selected patients $(<1 \%)$ undergoing general endotracheal anesthesia as determined necessary by the anesthesia team. All surgical procedures were performed by or under the direct supervision of a senior attending physician. All arthroplasties were performed through a modified Hardinge anterolateral approach or direct anterior approach with the patient in the supine position. The size of the components was determined on the basis of preoperative template measurements and intraoperative assessment. The size of the femoral head $(28,32$, or $36 \mathrm{~mm})$ varied on the basis of the size of the acetabular component, the type of liner (highly crosslinked polyethylene or ceramic), the age of the patient, and the preference of the surgeon. The desired position of the acetabular component was $15^{\circ}$ of anteversion. The femoral stem and head were inserted into place with neck length determined by evaluation of leg lengths intraoperatively. The abductor component was repaired with interrupted nonabsorbable sutures and fascia was mended with absorbable sutures in a similar fashion.

Patients who received a THA at our institution during the study period were given no traditional functional restrictions after their surgery, such as use of elevated seats, abduction pillows, and restriction from driving. Patients with left THA usually were allowed to start driving around 2 weeks after surgery, and those with right THA the third or fourth week postoperatively. All patients were given the freedom to use regular chairs and toilets and allowed to go up and down stairs when discharged home.

All patients were seen by a nurse practitioner and a physiotherapist during the first 2 weeks after surgery at home. They then were instructed to follow up at 6 weeks and at 6 months. Further followup was every 2 years and on an as-needed basis.

Followup visits were analyzed via attending office dictations reviewing the patient history, physical examination, imaging, physician impression, and plan of care. Dislocations were determined based on physician diagnosis. 
Two hundred two patients who were not able to return for followup were contacted by telephone and were asked whether they had any dislocation in the operated hip. Our primary outcome was dislocation at any point postoperatively.

Patients who dislocated were analyzed and several possible contributory factors, such as age, gender, BMI, diagnosis at the time of THA, comorbidities, and implant design (head size, offset), were investigated.

Three of us (CR, JP, RHR) evaluated all postoperative radiographs to determine cup inclination, version, and offset, as well as leg length discrepancy. The inclination of the cup was determined on the AP radiograph of the hip by measuring the angle that the long axis of the cup made with the horizontal line. The version of the acetabular component was determined from the lateral radiograph using previously described techniques [12]. Postoperative offset was measured on the AP view as the distance from the center of rotation of the femoral head to the axis of the femoral shaft at $90^{\circ}$ and was considered corrected if it was within $2 \mathrm{~mm}$ of the opposite side. Leg length correction was also measured on the AP view (distance from lesser trochanter to a horizontal line based on the teardrop or, if not visible, on the ischial tuberosity) and was considered corrected if it was within $5 \mathrm{~mm}$ of the other side [18]. All measurements made by the three observers were within two SDs of each other.

\section{Results}

Four dislocations occurred during this study at a mean time of 5 days (range, 3-12 days) after operation, leading to a dislocation rate of $0.15 \%$ (four of 2612 THAs). None of these dislocations were traumatic in origin (motor vehicle accident or high-energy trauma); all four were idiopathic. All dislocations occurred in patients with unilateral surgery. One idiopathic dislocation occurred in one patient (Patient A), who underwent THA for DDH with anatomic abnormality. Seven of the 2532 patients $(0.0023 \%)$ in this study underwent THA for DDH. Their mean age was lower than that of the total population (39.4 years versus
63.2 years). A second patient (Patient B) suffered a dislocation after getting off the toilet. This patient underwent THA after a failed subtrochanteric femoral fracture that progressed to hip osteoarthritis, requiring THA with a modular segmental prosthesis (Restoration ${ }^{\circledR}$ Modular System [RMS]; Stryker Orthopaedics, Mahwah, NJ) in the femoral component. Similarly, a third patient (Patient C) suffered two dislocations while getting off the toilet and getting out of bed. The indication for THA in this patient was end-stage osteoarthritis. Finally, a fourth dislocation occurred in a patient (Patient D) with no recollection of a specific mechanism. This patient's THA indication was also end-stage osteoarthritis. The surgical details of the four patients are displayed (Table 1). In these patients with dislocations, the BMI ranged from 20.3 to $35.9 \mathrm{~kg} / \mathrm{m}^{2}$, with an average of $24.5 \mathrm{~kg} / \mathrm{m}^{2}$. All were nonsmokers without history of diabetes or elevated blood glucose on admission. One patient with idiopathic dislocation had a medical history positive for both hypertension and hypothyroidism.

\section{Discussion}

We reported in 2005 how removal of functional restriction after primary THA performed through the anterolateral approach did not increase the incidence of dislocation. In this study, we expanded these findings by evaluating the incidence of early dislocation (within 6 months) after primary THA in a larger, uniformly non-restricted patient population.

We acknowledge limitations to our study. First, although one may argue the minimum followup of 6 months for this study was short [1], we believe the followup was sufficient to capture all dislocations that might have occurred as a result of not observing postoperative hip precautions; after all, no surgeon extends the postoperative hip precautions beyond 6 weeks. Second, 146 patients were lost to followup for the minimal requirement of 6 months. Although it is unlikely these patients sustained severe, unreducible dislocations, it is possible some could have dislocated and simply not reported it. Third, all operations were performed by senior surgeons at our institution who

Table 1. Surgical details of the four patients with dislocations

\begin{tabular}{lllll}
\hline Patient & Acetabular liner/femoral head & Femoral neck & Femoral stem* & Miscellaneous \\
\hline A & $28 \mathrm{~mm}$ & Standard & Accolade Size 2 & 13- x 203-mm RMS \\
B & $36 \mathrm{~mm}$ & Standard & $\begin{array}{c}\text { Nonunion of subtrochanteric } \\
\text { fracture }\end{array}$ & Accolade Size 3 \\
C & $36 \mathrm{~mm}$ & $5 \mathrm{~mm}$ & Accolade Size 3.5 & Draining wound \\
D & $32 \mathrm{~mm}$ & $4 \mathrm{~mm}$ & . & \\
\hline
\end{tabular}

* Femoral stems included Accolade ${ }^{\mathbb{R}}$ and Restoration ${ }^{\mathbb{R}}$ Modular System (RMS), both manufactured by Stryker Orthopaedics (Mahwah, NJ). 
have performed thousands of similar surgeries. Thus, it is possible our findings may not be generalizable to all THAs being performed using the anterolateral approach. Finally, we believe some patients, despite the lack of "mandated" hip precautions, may have implemented some restrictions for comfort purposes or fear of dislocation.

The risk of dislocation after THA is greatest during the first 3 months after operation [1, 5, 16, 21]. As a preventive measure and to protect soft tissue repair, many restrictions on patient activity and ROM have traditionally been put on patients during this time period [2,9]. Some of these guidelines include use of an abduction pillow in the early postoperative period, use of a pillow between the legs during sleep, use of high chairs and toilet seats, limiting flexion of the operative hip to less than $90^{\circ}$ and internal and external rotation to less than $20^{\circ}$, refraining from crossing legs, and avoiding automobile transportation as a driver or passenger to prevent excessive bending encountered during getting in and out. The rationale behind these precautions was to allow soft tissue healing around the newly replaced hip. Several studies have shown patient education is crucial to minimize hip dislocation after THA $[10,13]$. A recent study by Lubbeke et al. [7] reported a reduction in the risk of dislocation within 6 months after THA among participants in a preoperative education session involving a thorough review of hip precautions and instructions on how to adapt activities of daily living with those restrictions (eg, putting on shoes, getting in and out of chairs and the car).

Hip precautions can be a cause of discontent for the patients and could interfere with rehabilitation. They are associated with substantial economic and environmental burden to provide and discard the necessary equipment such as abduction pillows. The lack of a need for additional equipment and devices was associated with a cost savings of approximately US $\$ 655$ per patient. This included the cost for an abduction pillow (\$12), an elevated toilet seat (\$65), and an elevated chair (\$15/day to rent). This does not account for cost of transportation or loss of wages while away from work [14]. A few studies on removing hip precautions after primary THA reached the same conclusions (Table 2). Talbot et al. [19] prospectively followed 499 patients who underwent primary THA through the anterolateral approach. Patients observed no postoperative hip restrictions and no special devices were used. There were three dislocations $(0.6 \%)$ in the first 6 weeks. However, their study lacked a control group [19]. In our previous prospective study on 303 uncemented THA through the anterolateral approach, patients were randomized into two groups: restricted $(\mathrm{n}=152)$ and unrestricted $(\mathrm{n}=153)$. The restricted patients used abduction pillows, elevated toilet seats, and elevated chairs and avoided sleeping on the operated side and driving. At 6 months' followup, there were no dislocations in the unrestricted group. The patients

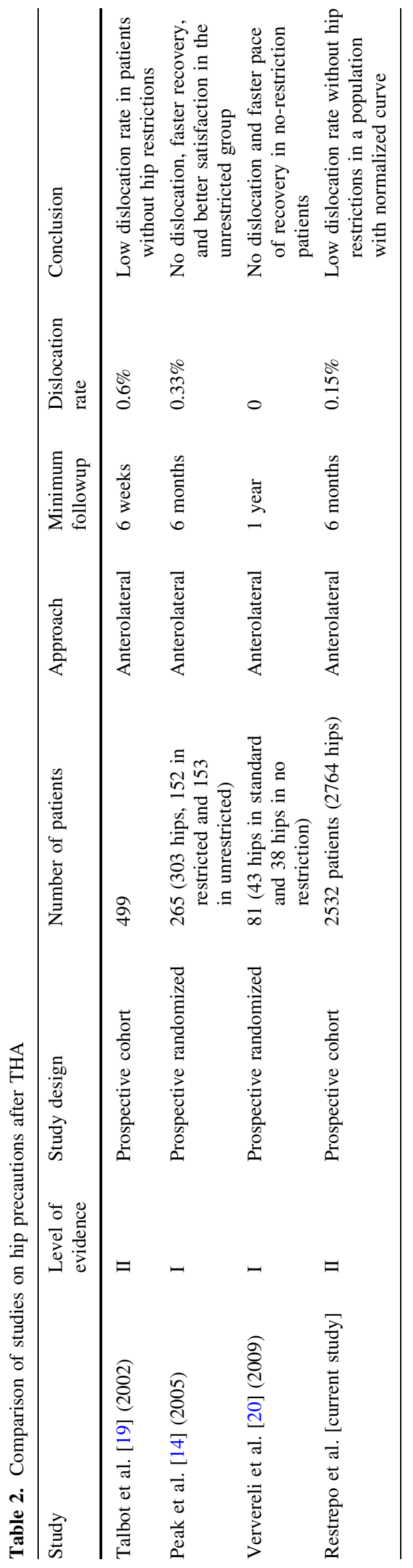


in the unrestricted group had faster return to normal activities and better satisfaction [14]. Ververeli et al. [20] reported on a similar randomized study on 81 patients divided into 43 patients receiving standard rehabilitation with restrictions and 38 patients receiving early rehabilitation without restrictions. All patients were operated on through the transgluteal approach. No incidents of dislocation occurred; however, patients in the early rehabilitation group were faster to ambulate with a cane, without a cane, and without a limp. They also drove earlier [20]. One potential argument with regard to the abovementioned studies could be that the lack of difference between restricted and nonrestricted groups was the result of a Type II statistical error. However, our study in a large cohort of patients undergoing primary THA, which found a $0.15 \%$ early dislocation rate, confirms the findings of those studies.

Hip dislocation after THA could occur as a result of multiple factors, including component malpositioning, soft tissue-related factors, and surgical approach. It is widely accepted there is an inherent increased risk for dislocation in the posterior approach because the majority of dislocations are posterior. Several studies have shown an anterolateral approach to THA has a lower rate of dislocation than a posterior approach due to its ease of access, superior visualization, and a predictable healing pattern $[8,11,17]$. Since all of the previous reported THAs were performed via the anterolateral approach, a no-restriction protocol can be applied only to patients undergoing THA via the anterolateral approach. However, there is increasing evidence that the rate of dislocation in the posterior approach would be comparable to that with anterior approaches if the posterior approach were augmented with an adequate soft tissue repair [6]. Additionally, previous studies have shown a correlation between larger femoral head and lower dislocation rates $[3,15]$. Although in our series two dislocations occurred with a 36-mm head, we could not perform a meaningful analysis evaluating the influence of femoral head size on dislocation because of the small number of patients with larger femoral head and the low incidence of dislocation. The other important issue, however, is that the size of the femoral head should be assessed with respect to the size of the acetabular component. Kelley [4] showed that the rate of dislocation was higher in patients with larger outer diameter of acetabular component paired to small diameter head size. Nevertheless, we believe, with improvement in soft tissue repair in the posterior approach and availability of larger femoral heads, no-restriction protocols may be considered for application to patients undergoing THA via the posterior approach.

This study revealed incidence of dislocation after THA in the absence of functional restrictions. This dislocation rate was similar to those of previous studies using patient precautions and is consistent with previous randomized prospective studies. We conclude removal of patient restrictions does not increase the incidence of early dislocation in primary THA through the anterior approaches.

\section{References}

1. Ali Khan MA, Brakenbury PH, Reynolds IS. Dislocation following total hip replacement. J Bone Joint Surg Br. 1981;63: 214-218.

2. Charnley J. Total hip replacement by low-friction arthroplasty. Clin Orthop Relat Res. 1970;72:7-21.

3. Hummel MT, Malkani AL, Yakkanti MR, Baker DL. Decreased dislocation after revision total hip arthroplasty using larger femoral head size and posterior capsular repair. J Arthroplasty. 2009;24(6 Suppl):73-76.

4. Kelley SS, Lachiewicz PF, Hickman JM, Paterno SM. Relationship of femoral head and acetabular size to the prevalence of dislocation. Clin Orthop Relat Res. 1998;355:163-170.

5. Khatod M, Barber T, Paxton E, Namba R, Fithian D. An analysis of the risk of hip dislocation with a contemporary total joint registry. Clin Orthop Relat Res. 2006;447:19-23.

6. Kwon MS, Kuskowski M, Mulhall KJ, Macaulay W, Brown TE, Saleh KJ. Does surgical approach affect total hip arthroplasty dislocation rates? Clin Orthop Relat Res. 2006;447:34-38.

7. Lubbeke A, Stern R, Garavaglia G, Zurcher L, Hoffmeyer P. Differences in outcomes of obese women and men undergoing primary total hip arthroplasty. Arthritis Rheum. 2007;57: 327-334.

8. Mallory TH, Lombardi AV, Jr., Fada RA, Herrington SM, Eberle RW. Dislocation after total hip arthroplasty using the anterolateral abductor split approach. Clin Orthop Relat Res. 1999;358:166-172.

9. Masonis JL, Bourne RB. Surgical approach, abductor function, and total hip arthroplasty dislocation. Clin Orthop Relat Res. 2002;405:46-53.

10. Morrey BF. Difficult complications after hip joint replacement. Dislocation. Clin Orthop Relat Res. 1997;344:179-187.

11. Moskal JT, Mann JW, 3rd. A modified direct lateral approach for primary and revision total hip arthroplasty. A prospective analysis of 453 cases. J Arthroplasty. 1996;11:255-266.

12. Murray JE. Orientation-specific effects in picture matching and naming. Mem Cognit. 1999;27:878-889.

13. Paterno SA, Lachiewicz PF, Kelley SS. The influence of patientrelated factors and the position of the acetabular component on the rate of dislocation after total hip replacement. J Bone Joint Surg Am. 1997;79:1202-1210.

14. Peak EL, Parvizi J, Ciminiello M, Purtill JJ, Sharkey PF, Hozack WJ, Rothman RH. The role of patient restrictions in reducing the prevalence of early dislocation following total hip arthroplasty. A randomized, prospective study. J Bone Joint Surg Am. 2005; 87:247-253.

15. Peters CL, McPherson E, Jackson JD, Erickson JA. Reduction in early dislocation rate with large-diameter femoral heads in primary total hip arthroplasty. J Arthroplasty. 2007;22(6 Suppl 2):140-144.

16. Phillips CB, Barrett JA, Losina E, Mahomed NN, Lingard EA, Guadagnoli E, Baron JA, Harris WH, Poss R, Katz JN. Incidence rates of dislocation, pulmonary embolism, and deep infection 
during the first six months after elective total hip replacement. J Bone Joint Surg Am. 2003;85:20-26.

17. Ritter MA, Harty LD, Keating ME, Faris PM, Meding JB. A clinical comparison of the anterolateral and posterolateral approaches to the hip. Clin Orthop Relat Res. 2001;385:95-99.

18. Sayed-Noor AS, Hugo A, Sjoden GO, Wretenberg P. Leg length discrepancy in total hip arthroplasty: comparison of two methods of measurement. Int Orthop. 2009;33:1189-1193.
19. Talbot NJ, Brown JH, Treble NJ. Early dislocation after total hip arthroplasty: are postoperative restrictions necessary? J Arthroplasty. 2002;17:1006-1008.

20. Ververeli PA, Lebby EB, Tyler C, Fouad C. Evaluation of reducing postoperative hip precautions in total hip replacement: a randomized prospective study. Orthopedics. 2009;32:889-893.

21. Woo RY, Morrey BF. Dislocations after total hip arthroplasty. J Bone Joint Surg Am. 1982;64:1295-1306. 